\title{
O que esperar da Selic e do câmbio nominal nos próximos meses? Uma visão pelo modelo VAR com restrição
}

\author{
Luciano D'Agostini* \\ José Luis Oreiro
}

Segundo a Ata da última reunião do Copom, divulgada em 16 de março, interpretamos que as projeções da taxa de câmbio nominal e da taxa Selic, esperadas para os próximos meses, permanecem com as mesmas trajetórias observadas há meses: apreciação do real e quedas graduais para a taxa Selic (atualmente em 16,5\% a.a.).

Diante deste cenário traçado pelo Banco Central do Brasil (BCB), faremos pelo método de Vetores-Auto Regressivos (VAR) as previsões das taxas de câmbio nominal e Selic para os próximos meses.

Antes, cabe aqui mostrar a trajetória de algumas variáveis que acreditamos serem relevantes, tanto da economia norte-americana, quanto da economia brasileira, que podem explicar o comportamento da apreciação do real em relação ao dólar ${ }^{13}$.

Pelo lado da economia americana, destacam-se: (i) a queda de 20,5\% das reservas internacionais desde dezembro de 2004 (de US \$ 90,1 bilhões para US\$ 71,6 bilhões em fevereiro de 2006); (ii) desde julho de 2004 são observados sucessivos aumentos das taxas de juros dos títulos do tesouro norte-americano (2,25\% a.a. em julho de 2004 para 4,75\% a.a. em março de 2006); (iii) inflação mais alta e; (iv) crescentes déficits orçamentários.

Pelo lado da economia brasileira, destacam-se: (i) a política de recomposição das reservas internacionais, que saltou de US $\$ 35,8$ bilhões em 2002 para US $\$ 59,8$ bilhões no final de março de 2006 (um aumento de 67\%), mesmo após a liquidação antecipada de US\$ 15,5 bilhões referente à dívida junto ao Fundo Monetário Internacional (FMI), recompra de títulos de dívida soberana e o anúncio do exercício da opção de compra dos títulos bradies; (ii) isenção para a aplicação de estrangeiros em títulos públicos federais no mercado doméstico, inclusive sem pagamento de CPMF, que contribuiu para a entrada de dólares no país; (iii) sucessivos superávits comerciais; (iv) geração de superávits primários; (v) a melhora no perfil da dívida pública interna, tanto no que se refere à queda do componente ligado à taxa de

\footnotetext{
* Doutorando em Desenvolvimento Econômico pela Universidade Federal do Paraná (UFPR). Professor Assistente da Faculdade Radial, Camões e do IBPEX. Endereço eletrônico:lucianodagostini@yahoo.com.br.

** Doutor em Economia (IE/UFRJ). Professor do Departamento de Economia da UFPR e pesquisador do CNPq. Endereço eletrônico: joreiro@ufpr.br. Página pessoal: http://www.joseluisoreiro.ecn.br.

${ }^{13}$ Em junho de 2004, US\$1,00 = R \$3,12. Em março de 2006 , US\$1,00 = R $\$ 2,14$ (queda de 31\%).
} 
câmbio, quanto no aumento de sua parcela atrelada a índices de preços; (vi) o controle da inflação e a política monetária restritiva, com taxas nominais de juros altas.

No mais, o BCB, por intermédio do sistema de Metas de Inflação, tem sob sua responsabilidade manter a taxa de inflação sob controle. Este sistema, segundo o BCB, faz da taxa de juros Selic o principal instrumento de política monetária para alcançar o objetivo proposto $^{14}$. Por isso, a fixação das taxas de juros em cada reunião do Copom (agora de $45 \mathrm{em}$ 45 dias) se apóia na previsão de inflação.

Sabe-se que a meta de inflação para 2006 é de 4,5\% a.a. e de 4\% a.a. para 2007. O IPCA acumulado no ano de 2006 (janeiro e fevereiro) está em 1\%. As expectativas do BCB quanto à variação do IPCA para 2006, de acordo com a Ata da $117^{a}$ reunião do Copom, divulgada em 16/03/2006, mostram-se alinhadas com a meta de 4,50\% a.a., estabelecida pelo Conselho Monetário Nacional (CMN).

Cruzando as informações da Ata $n^{\circ} 117$ com notas de reuniões anteriores ( $\mathrm{n}^{\text {os }}$ 116, 115 e 114), o Copom afirma que a sua posição cautelosa da política monetária tem sido fundamental para aumentar a probabilidade de convergência da inflação para a trajetória de metas. Dessa forma, a flexibilização gradual da política monetária via Selic (na média 0,75 p.p. a cada 45 dias) parece ser mais uma medida de cautela perante novos surtos inflacionários do que a própria margem de queda das taxas de juros que o BCB já poderia efetuar, como uma queda, por exemplo, de 1 p.p. ${ }^{15}$.

Por outro lado, as decisões de política monetária dos últimos meses também contribuem de forma importante para aumentar as expectativas dos agentes pela queda dos juros reais nos próximos meses.

Assim, diante do objetivo da política monetária de manter a inflação sob controle, mesmo com queda gradual da Selic, aliado ao aumento de reservas cambiais e do cenário da economia americana (elevação das taxas de juros e queda de reservas), o que acontecerá com a taxa de câmbio nominal nos próximos meses?

Nestes cenários, pelo modelo de Vetores Auto-Regressivos (VAR), o objetivo é prever: (i) a taxa de câmbio nominal e; (ii) a taxa de juros Selic, esperadas para os próximos 2 e 4 meses, respectivamente.

\footnotetext{
14 Para detalhes ver: D’Agostini, L.; Oreiro, J.L. "Prevendo o comportamento de curto-prazo da taxa de juros Selic a partir de um modelo VAR com restrição". Economia \& Tecnologia, Ano. 01, Vol. 02, jul./ago. 2005. Fonseca, M. W.; Oreiro, J.L. "Mecanismos de transmissão da política monetária e controle de inflação no Brasil”. Economia \& Tecnologia, Ano. 01, Vol. 03, set./dez. 2005.

156 membros votaram a favor da queda de 0,75 p.p. e 3 membros votaram pela queda de 1 p.p. da Selic.
} 
A taxa Selic será determinada com base na avaliação dos valores correntes e passados de um certo conjunto de variáveis, a saber: produto, moeda, câmbio e preços. No exercício de estimação da taxa de câmbio nominal incluiremos também as reservas internacionais do Brasil e dos Estados Unidos e a taxa de juros norte-americana. Portanto, para a previsão da Selic utilizam-se 5 variáveis e para o câmbio nominal, utilizam-se 8 variáveis.

Assumindo endogeneidade em todas as variáveis inseridas no sistema, a taxa de câmbio nominal e a taxa Selic podem ser estimadas consistentemente pelo VAR. Os procedimentos econométricos usados são resumidos nas seguintes etapas ${ }^{16}$ : (i) logaritimizar as séries; (ii) efetuar o teste de raiz unitária pelo ADF; (iii) efetuar os testes de Cointegração de Johansen-Juselius (JJ); (iv) escolher a defasagem ótima do VAR pelo critério de Akaike (AIC) e Schwartz (SBC); (v) conforme o teste JJ adota-se o modelo VAR simples ou com correção de erro (VECM); (vi) determinar os coeficientes das equações por MQO; (vii) observar a equação de equilíbrio de longo prazo entre as variáveis, dado pelo VECM; (viii) substituir os valores presentes e passados na equação da taxa de câmbio e na equação da taxa de juros.

Os modelos VAR são do tipo Log-Log. Isto faz com que os coeficientes dos parâmetros estimados sejam interpretados como elasticidades da taxa de juros com respeito às variáveis do sistema (equação 1) e elasticidades da taxa de câmbio nominal com respeito às variáveis do sistema (equação 2).

Efetuando as substituições dos valores das variáveis defasadas no sistema, para diversos períodos de tempo, temos o valor das elasticidades dos juros e do câmbio. Estas elasticidades, por sua vez, determinam, para os períodos $t+1, t+2, \ldots, t+n$, os valores das previsões das taxas de juros subseqüentes.

Por questões probabilísticas do modelo, quanto maior é o tempo de previsão, menor é a possibilidade de acerto. Portanto, o modelo VAR serve apenas para previsões de curto prazo. Por isso, nas tabelas 1 e 2, mostram-se os valores esperados do câmbio nominal e da Selic para 2 e 4 períodos à frente, respectivamente.

Inclui-se no modelo VAR, dados mensais de janeiro de 1999 a fevereiro de 2006, das seguintes variáveis: para preços, utilizou-se o IPCA do IBGE, por ser a taxa de inflação utilizada para estabelecer a meta de inflação; para a taxa básica de juros, utilizou-se a Selic nominal, que é a taxa de juros pelo qual o BCB tenta controlar a inflação; para o produto

\footnotetext{
16 Para detalhes dos procedimentos metodológicos, ver: D’Agostini (2004). Monismo versus Não-monismo no Brasil (1994-2004): uma abordagem econométrica por vetores auto-regressivos com restrição. Dissertação de Mestrado, nov. 2004, 143p, Curitiba: UFPR.
} 
utiliza-se o PIB mensal em valores correntes, medida em milhões de reais. Foram utilizadas ainda a série de moeda $\mathrm{M}_{2}$, a série de câmbio nominal e a série de reservas internacionais brasileiras, pelo conceito de liquidez total, extraídos do BCB. Por fim, a taxa de juros norteamericana, que é a taxa de desconto do "Banco Central dos Estados Unidos", e o nível de reservas americanas foram extraídos do Federal Reserve e do FMI, respectivamente.

Os resultados da previsão da taxa de câmbio nominal, estimado pelo VAR(4) com correção de erros estão dispostos na tabela 1, enquanto que a previsão da taxa de juros, estimada por um modelo VAR(5) com correção de erros está na tabela 2.

Tabela 1. Previsão da taxa de câmbio nominal (US\$/R\$) pelo VAR

\begin{tabular}{l|c|c|c}
\hline \multicolumn{1}{c|}{ Mês } & $\begin{array}{c}\text { Câmbio } \\
\text { Efetivo }\end{array}$ & $\begin{array}{c}\text { Previsão Câmbio } \\
\text { Nominal** }\end{array}$ & $\begin{array}{c}\text { Elasticidade } \\
\text { Câmbio }\end{array}$ \\
\hline Março/2006 & $2,1421^{*}$ & - & -0.00402388490313 \\
Abril/2006 & - & 2,1265 & -0.0031714765463 \\
Maio/2006 & - & 2,1197 & - \\
\hline
\end{tabular}

Fonte: Banco Central do Brasil e dados da pesquisa.

Notas: * 2,1421 corresponde à média do câmbio até 28 de março. **O erro de previsão é 3\%.

Tabela 2. Comparação da taxa Selic efetiva com a taxa Selic estimada em \% a.a.

\begin{tabular}{l|c|c|c}
\hline \multicolumn{1}{c|}{ Mês } & $\begin{array}{c}\text { Selic efetiva } \\
\text { \% a.a. }\end{array}$ & $\begin{array}{c}\text { Previsão da } \\
\text { Selic }\end{array}$ & $\begin{array}{c}\text { Elasticidade } \\
\text { Juros }\end{array}$ \\
\hline Março/2006 & $16,50 \%$ & - & -0.0229036953991 \\
Abril/2006 & - & $16,10 \%$ & -0.0304087862953 \\
Maio/2006 & - & $15,56 \%$ & -0.0346403443425 \\
Junho/2006 & - & $15,02 \%$ & -0.0357336306673 \\
Julho/2006 & - & $14,47 \%$ & - \\
\hline
\end{tabular}

Fonte: Banco Central do Brasil e dados da pesquisa.

Os resultados da tabela 1 apontam para a continuidade do processo de apreciação da taxa nominal de câmbio nos próximos meses. Com efeito, as previsões do modelo apontam para uma taxa nominal de câmbio próxima de $\mathrm{R} \$ 2,10$ para o mês de maio do corrente ano. Para o mês de abril o modelo indica a previsão da Selic em 16,10\%. Como a taxa básica de juros da economia brasileira está atualmente em 16,5\% ao ano, segue-se que nossa previsão é que na próxima reunião do Copom, a ser realizada nos dias 18 e 19 de abril, ela será reduzida em apenas 0.5 ponto percentual, sendo fixada em $16 \%$ ao ano. Dessa forma, o modelo VAR aqui apresentado prevê uma redução do ritmo de queda da taxa básica de juros nas próximas reuniões do Copom. 


\section{Anexos}

A equação (1) de previsão da taxa de juros simulada é:

$\mathrm{D}(\mathrm{LSELIC})=-0.02018059459 *($ LSELIC $(-1)+0.585066784 *$ LM2(-1) + 1.739243972*LTXC(-1) 1.302373288*LIPCA(-1) - 0.4484326925*LPIB(-1) - 0.01201685552*(@TREND(99:02)) - 11.07871588) + $0.5135735757 * \mathrm{D}(\operatorname{LSELIC}(-1)) \quad-\quad 0.0335545465 * \mathrm{D}(\operatorname{LSELIC}(-2)) \quad+\quad 0.0839319956 * \mathrm{D}(\operatorname{LSELIC}(-3)) \quad-$ $0.1267196875 * \mathrm{D}(\operatorname{LSELIC}(-4)) \quad+\quad 0.01513291264 * \mathrm{D}(\operatorname{LSELIC}(-5)) \quad-\quad 0.1015569921 * \mathrm{D}(\mathrm{LM} 2(-1)) \quad+$ $0.03867093221 * \mathrm{D}(\mathrm{LM} 2(-2))+0.1878129845 * \mathrm{D}(\mathrm{LM} 2(-3)) \quad+\quad 0.3268337047 * \mathrm{D}(\mathrm{LM} 2(-4)) \quad+$ $0.2479503308 * \mathrm{D}(\operatorname{LM} 2(-5)) \quad+\quad 0.1094628886 * \mathrm{D}(\operatorname{LTXC}(-1)) \quad-\quad 0.00201962325 * \mathrm{D}(\operatorname{LTXC}(-2)) \quad+$ $0.1562505529 * \mathrm{D}(\operatorname{LTXC}(-3))+0.05962606285 * \mathrm{D}(\operatorname{LTXC}(-4))+0.1119794155 * \mathrm{D}(\operatorname{LTXC}(-5)) \quad-$ $0.02169661577 * \mathrm{D}(\operatorname{LIPCA}(-1)) \quad-\quad 0.01497849278 * \mathrm{D}(\mathrm{LIPCA}(-2)) \quad$ - $\quad 0.01301846577 * \mathrm{D}(\mathrm{LIPCA}(-3)) \quad$ $0.008815632698 * \mathrm{D}(\operatorname{LIPCA}(-4)) \quad-\quad 0.004705401764 * \mathrm{D}(\operatorname{LIPCA}(-5)) \quad+\quad 0.0007469799986 * \mathrm{D}(\mathrm{LPIB}(-1)) \quad-$ $0.02409585669 * \mathrm{D}(\mathrm{LPIB}(-2))+0.03614985971 * \mathrm{D}(\mathrm{LPIB}(-3)) \quad-\quad 0.053882506 * \mathrm{D}(\mathrm{LPIB}(-4)) \quad$ $0.03884086278 * \mathrm{D}(\operatorname{LPIB}(-5))-0.01108734511+6.450438595 \mathrm{e}-05^{*}(@ \operatorname{TREND}(99: 02))$

A equação (2) de previsão da taxa de câmbio nominal simulada é:

$\mathrm{D}(\mathrm{LTXC})=-0.1194549622 *($ LSELIC $(-1)-0.1136304566 * \operatorname{LRBR}(-1)+0.5079931725 * \operatorname{LREUA}(-1)+$ 0.6564356466*LJEUA(-1) + 1.380676173*LTXC(-1) - 0.00645716451*(@TREND(99:02)) - 9.43185342 ) 0.03577534024*( LM1(-1) - 0.08617191365*LRBR(-1) + 0.6203690753*LREUA(-1) - 0.1157641509*LJEUA(-1) - 1.253437871*LTXC(-1) - 0.01009159295*(@TREND(99:02)) - 23.04143618 ) + 0.2444572232*( LM2(-1) 0.1423713521*LRBR(-1) + 0.2639001768*LREUA(-1) + 0.1103187075*LJEUA(-1) + 0.3350072954*LTXC(-1) - 0.0120374908*(@TREND(99:02)) - 21.13382695 )- 0.05249511076*(LIPCA(-1) + 1.87226279*LRBR(-1) $4.115276627 * \operatorname{LrEUA}(-1)+1.524581422 * \operatorname{LJEUA}(-1)+\quad+\quad 8.403957808 * \operatorname{LTXC}(-1)$ 0.02881135805*(@TEND(99:02)) + 21.03601263 ) - 0.5070805464*(LPIB(-1) - 0.06643849152*LRBR(-1) + $0.2418436291 * \operatorname{LREUA}(-1) \quad$ - $\quad 0.1378206154 * \operatorname{LJEUA}(-1) \quad$ - $\quad 0.7021405664 * \operatorname{LTXC}(-1) \quad$ 0.007832806989*(@TREND(99:02)) - $12.71441512 \quad$ + $0.1717010193 * \mathrm{D}(\operatorname{LSELIC}(-1)) \quad+$ $0.1250562329 * \mathrm{D}(\operatorname{LSELIC}(-2)) \quad+\quad 0.04595733818 * \mathrm{D}(\operatorname{LSELIC}(-3)) \quad-\quad 0.122766074 * \mathrm{D}(\operatorname{LSELIC}(-4)) \quad+$ $0.07765952504 * \mathrm{D}(\mathrm{LM} 1(-1))+0.1192551262 * \mathrm{D}(\mathrm{LM} 1(-2))-0.160596311 * \mathrm{D}(\mathrm{LM} 1(-3))-0.2198837624 * \mathrm{D}(\mathrm{LM} 1(-$ 4)) - $0.5879193523 * \mathrm{D}(\mathrm{LM} 2(-1)) \quad-\quad 0.9108408611 * \mathrm{D}(\mathrm{LM} 2(-2)) \quad+\quad 0.2115702555 * \mathrm{D}(\mathrm{LM} 2(-3)) \quad+$ $0.130477662 * \mathrm{D}(\mathrm{LM} 2(-4))+0.04309122901 * \mathrm{D}(\operatorname{LIPCA}(-1))+0.02113457855 * \mathrm{D}(\operatorname{LIPCA}(-2))+$ $0.0211320575 * \mathrm{D}(\operatorname{LIPCA}(-3))+0.002307140499 * \mathrm{D}(\operatorname{LIPCA}(-4))+0.1498618892 * \mathrm{D}(\operatorname{LPIB}(-1))+$ $0.3611840819 * \mathrm{D}(\operatorname{LPIB}(-2))+0.3757389635 * \mathrm{D}(\operatorname{LPIB}(-3))+0.1396865235 * \mathrm{D}(\operatorname{LPIB}(-4))+$ $0.20064735 * \mathrm{D}(\operatorname{LRBR}(-1))+0.2163337878 * \mathrm{D}(\operatorname{LRBR}(-2))+0.3362292987 * \mathrm{D}(\operatorname{LRBR}(-3))+$ $0.1974906148 * \mathrm{D}(\operatorname{LrBR}(-4)) \quad-\quad 0.1182461034 * \mathrm{D}(\operatorname{LREUA}(-1)) \quad+\quad 0.1944800271 * \mathrm{D}(\operatorname{LrEUA}(-2)) \quad$ $0.2908677722 * \mathrm{D}(\operatorname{LrEUA}(-3))+0.2339676474 * \mathrm{D}(\operatorname{LREUA}(-4))+0.06835584334 * \mathrm{D}(\mathrm{LJEUA}(-1))+$ $0.1032288167 * \mathrm{D}(\mathrm{LJEUA}(-2)) \quad-\quad 0.01202356144 * \mathrm{D}(\mathrm{LJEUA}(-3)) \quad+\quad 0.01707870149 * \mathrm{D}(\mathrm{LJEUA}(-4)) \quad+$ $0.05045260276 * \mathrm{D}(\mathrm{LTXC}(-1)) \quad-\quad 0.3599400511 * \mathrm{D}(\mathrm{LTXC}(-2)) \quad$ - $\quad 0.03355411529 * \mathrm{D}(\operatorname{LTXC}(-3)) \quad$ 0.1394890142*D(LTXC(-4)) + 0.02513324448 - 0.0005550875032*(@TREND(99:02))

Onde $\mathrm{D}($.$) é a primeira diferença; (-1), (-2)..., (-5) são as defasagens das variáveis utilizadas no modelo e;$ @TREND (.) é o componente de tendência. 
